\title{
Fusion 2050 - European and Polish Perspective
}

\author{
Ryszard S. Romaniuk
}

\begin{abstract}
Fusion, in all its varieties, is a very current subject of science and technology. The results of strongly exothermic reaction of thermonuclear fusion between nuclei of deuterium and tritium are: helium nuclei and neutrons, plus considerable kinetic energy of neutrons of over $14 \mathrm{MeV}$. DT nuclides synthesis reaction is probably not the most favorable one for energy production, but is the most advanced technologically. More efficient would be possibly an aneutronic fusion. The EU by its EURATOM agenda prepared a Road Map for research and implementation of Fusion as a commercial method of thermonuclear energy generation in the time horizon of 2050. The milestones on this road are tokomak experiments JET, ITER and DEMO, and neutron experiment IFMIF. There is a hope, that by engagement of the national government, and all research and technical fusion and plasma communities, part of this Road Map may be realized in Poland. The infrastructure build for fusion experiments may be also used for material engineering research, chemistry, biomedical, associated with environment protection, power engineering, security, etc. Construction of such research and industrial accelerator and tokomak infrastructure may have potentially a profound meaning for the development of science and technology in Poland.
\end{abstract}

Keywords-European research programs, 7FP, accelerator science and technology, large research infrastructures, JET, ITER, IFMIF, DEMO, nuclear fusion, thermonuclear power engineering, DT fusion, neutrons, aneutronic technologies, inertial fusion, tokomak, stellarator, fuser, fusion reactor, tritium, electronics for fusion

\section{INTRODUCTION - FUSION ROAD MAP IN EUROPE}

$\mathbf{P}$ ROFESSOR Pierre-Gilles de Gennes said, during his Nobel Lecture in physics in 1991 about the thermonuclear power, the following words: "We say that we close the Sun in a box. Brilliant idea, however, we do not know how to build this box". The thermonuclear reaction is the main, apart from gravitational energy, power source of a star. A controlled thermonuclear reaction, is one of promising, prospective, safe and clean energy sources for the humankind, especially after exhausting the fossil fuels. In the European Union Strategy Plan, concerning the development of power technologies, there is expressed an urgent necessity to intensify and stabilize works in the direction to assure production of electrical power from nuclear fusion in Europe till the year 2050. Fusion Road Map for Europe (FRME), in agreement with the European Commission, was prepared and is constantly updated by the European Fusion Development Agreement - EFDA. The EFDA Organization [1] provides international framework of educational [2], research and technical, as well as political efforts for the future energy sources. EFDA Agreement was signed by 28 European countries, including Poland. The EFDA is a part of the European Union EURATOM Association [3]. The European Atomic Energy Community EURATOM is

R. S. Romaniuk is with Warsaw University of Technology, Poland (e-mail: rrom@ise.pw.edu.pl). now under transformation from Association into the European Consortium. The aim of all these organizational and technical activities is to make from fusion a credible, efficient, and cost-effective energy source. The assumed method is strict realization of the prepared Fusion Road Map in Europe. The first stage of the work was defined in the Program Perspective of the EC "Horizon 2020". Fusion Road Map was preceded with numerable analyses carried out since 2000 and experiments such as Joint European Torus - JET started from the end of seventies of the 20tieth Century. The milestones on the European Fusion Road are: JET [4] - which is a common experimental tokomak located in Culham, near Oxford in the UK, ITER [5] (International Thermonuclear Experimental Reactor) tokomak located in Cadarache in France, IFMIF (International Fusion Material Irradiation Facility), and DEMO (Demonstration - Test Thermonuclear Power Plant). A key role in this road is played by ITER experiment.

Polish research and technical fusion community prepares itself to participate in these global undertakings by integrating and consolidating the efforts, and searching for financial and political support. A conference was organized in May 2013 on "The Future of Fusion Research in Poland" [6]. The key players of these activities in Poland are: IFPiLM, POLATOM, IFJ, IFD-UW, NCBJ, and PW. The paper tries to summarize state-of-the-art of fusion technology in Europe and Poland, emphasizing this country chances to participate actively in the global endeavors. This paper was presented during the WILGA 2013 Symposium on Electronics for HEP Experiments, FEL lasers, Fusion and Astroparticle Physics [7], [8] and was published in Polish in Elektronika [9]. Participation in global fusion program is a strong enabler for local research and industry.

\section{Aneutronic And Neutronic Fusion}

It is necessary to build in Europe (or internationally) an intense source of fast neutron beam of $14 \mathrm{MeV}$ kinetic energy - IFMIF - International Fusion Materials Irradiation Facility [10]. This source is to be used for doing the research on materials for DT fusion environment. The DEMO (Test Thermonuclear Power Plant) experiment will be a single and only one milestone on the road to a commercial thermonuclear power plant. Material engineering research with neutron beams are necessary. The Deuterium - Tritium (DT) reaction is highly neutronic. The neutrons originating from this fusion reaction possess $14 \mathrm{MeV}$ of kinetic energy. Lowneutron reactions (aneutronic fusion) require much more difficult conditions to be reached, i.e. higher temperatures and pressures in the plasma. Aneutronic fusion is defined when the amount of energy carried by the neutrons, from the global released energy during the fusion reaction, is not higher 
than $1 \%$. Neutrons in the DT fusion reaction carry $80 \%$ of the total energy. The DT reaction unfolds in the following way: ${ }^{2} \mathrm{H}+{ }^{3} \mathrm{H} \rightarrow(\mathrm{n}+14,1 \mathrm{MeV})+\left({ }^{4} \mathrm{H}+3,5 \mathrm{MeV}\right)$. During such an exothermic reaction, there is released kinetic energy of products and gamma radiation. This energy is next dissipated in the vicinity of the reaction spot. This energy has to be received from this area and converted to electrical energy.

The released energy is proportional to the mass deficit in the reaction. The mass deficit in such reactions, also with other atoms than $\mathrm{D}$ and $\mathrm{T}$, up to iron $\mathrm{Fe}$ is negative. Atom nuclei (nuclides) of other elements have different nucleon (proton and neutron) binding energies. The biggest binding energy per a single nucleon has Fe. In consequence, for heavier elements, the exothermic reaction is not fusion but fission. Thus, the nuclear power industry uses uranium as a fuel. The fusion of light nuclei, positively charged, is possible after overcoming the electrostatic potential barrier (repelling Coulomb force). The reacting nuclei have to possess relevant kinetic energy, obtained by heating or in accelerators. Fusion is accomplished when the nuclei go as close to each other where the strong attracting nuclear force overcomes the repelling electrostatic force.

There are not checked theories about so called cold fusion of nuclides (electrolytic, palladium - zirconium, nickel - hydrogen, sonofusion, piroelectrical, magnetic, spontaneous, etc.).

Aneutronic reactions of the biggest cross-section are, for example: $\mathrm{D}+{ }^{3} \mathrm{He} \rightarrow\left({ }^{4} \mathrm{He}+3,6 \mathrm{MeV}\right)+(\mathrm{p}+14,7 \mathrm{MeV})$, or with participation of lithium $\mathrm{D}+{ }^{6} \mathrm{Li} \rightarrow 2{ }^{4} \mathrm{He}+22,4 \mathrm{MeV}$, and also other ones with participation of $\mathrm{Li}, \mathrm{He}$ and $\mathrm{B}$ nuclei. There are carried out research works on aneutronic fusion, using Polywell method, in a machine called a fuser, and in particular in the $\mathrm{Z}$ machine constructed at Nat. Sandia Laboratory in Albuquerque. Potentially, the fuser might have simpler construction from a tokamak, machines used in JET or ITER. Polywell combines magnetic and inertial electrostatic methods of plasma confinement, in order to obtain conditions for a controlled thermonuclear synthesis. The configuration of fields keeps the electrons inside the fusion reactor, what generates nearly spherical and large negative electrical potential used for acceleration and confinement of ions subject to nuclear synthesis. Machine $\mathrm{Z}$, which is a strong generator of Xrays, and a compressor of hot matter, is tested for the MagLIF method or Magnetized Linear Inertial Fusion. Search for aneutronic fusion stems simply from the issues with strongly penetrating neutronic radiation. Neutronic radiation causes strong damage, and leads to neutronic activation of numerable materials - the materials turn to radiative. It is necessary to use screens, and management of the experiment from distant locations.

Neutron radiation does not lead directly to generation of electricity. There are needed intermediate energy conversion stages. In the case of radiation with charged particles, direct electricity generation is possible. Aneutronic fuel cycle (like D-He, D-Li, He-Li, He-He, p-Li, p-B, p-N), as differentiated from the DT cycle, requires much more difficult technical reaction conditions. Some of them still cannot be fulfilled by the current status of technology.

\section{INERTIAL FUSION}

An alternative to fusion with magnetic confinement is fusion with inertial confinement. Inertial fusion with laser triggered ignition is tested in the NIF experiment in LLNL USA [11]. A laser generates shock wave in the fuel. NIF uses DT fuel, which is heated during ps time, with impulse power of over $1 \mathrm{PW}$, to temperature $10^{9} \mathrm{~K}$ and compresses to the density of $10^{6} \mathrm{~kg} / \mathrm{m}^{3}$. Based on the experiences of NIF the next facility is built LIFE (Laser Inertial Fusion Energy) [12], which may work with much bigger ignition frequency, over $10 \mathrm{~Hz}$ or more. NIF gives a single pulse per a few hours. Now, there is observed fast development of the research on inertial confinement fusion. The leading centers are NIF/USA and ELI and HIPER and LMJ in Europe. Heated external layer of the container with the DT fuel explodes outward generating shock wave inward, which compresses contents of the target. Construction of the target may be simple - a glass sphere with DT fuel, or complex, so called hohlraum cavity with laser generation of intermediate X-ray wave inside the cavity. Shock wave, thermal and pressure, at sufficient energy and relevant target geometry, of $10 \mathrm{mg}$ in mass, should cause thermonuclear ignition. Energy equivalent of this target burning is equal to burning immediately a single oil barrel of 250 liters. Till now, the experiments of concern did not achieve stable thermonuclear burning of target, with considerable excess of outgoing energy over the incoming energy into the reaction chamber. Development of this idea is fast fusion, where during the exact moment of the biggest fuel compression exactly into this point hits a femtosecond and petawatt laser pulse. Alternatively this point may be hit by a sub-relativistic (or at least very fast) material microobject - a projectile of very large kinetic energy. Today the costs of potential commercial energy from fusion reactions are not very promising. Additionally, fusion awakes doubts because of close relations with the research on compact fusion weapons. Despite that, inertial fusion has large development potential, due to production of much more neutrons, even several orders of magnitude, than during the Spallation reaction exercised in such infrastructures like Spallation Neutron Source - SNS/USA [13], or European Spallation Source ESS [14] in Lund, Sweden. Also, there are researched ideas of hybrid nuclear generation of electrical energy in the FF process - Fusion-Fission. This research is carried out inside the LIFE project in LLNL.

\section{Pragmatic Approach to Fusion Energy in EUROPE AND POLAND}

European R\&D Fusion Community has decided that the ITER will be a key element of the road map leading to commercial fusion energy. The experience gained during the exploitation of the JET machine are directly adapted to ITER experiment. It is expected that ITER will reach the majority of the assumed and most important research and technical aims. These achievements will be the basis for a decision of building an experimental demonstration thermonuclear plant called DEMO. Such a critical date of the most important parameters review was set for 2020. Even more important date on the road map is 2030. This year was scheduled preliminarily as the 
decision year for the beginning of the infrastructure building leading to DEMO. The DEMO infrastructure should be initiated in 2030 if only Europe is going to be prepared till 2050 to take a decision on wide commercialization of thermonuclear energy. The decision in 2050, concerning exploitation thermonuclear plant, will be taken based on the experiences with exploitation of DEMO. Till this time a great number of research, development, technical, financial and social problems have to be resolved. ITER reaction is direct fusion of DT fuel, thus, it is necessary to turn community attention on safety issues combined with kinetic neutron radiation, and concerning generally building and exploitation of thermonuclear plants.

The consequence of focusing the research and technical efforts around ITER is also strong financial focusing proposed in the European road map for fusion on this experiment, and in particular preparing the ITER for efficient exploitation. No additional resources for strengthening ITER were assumed, i.e. for building of infrastructure and commissioning. It means that ITER should be built in accordance with the original timetable and within the approved budget. The fusion experiment ITER, standing at the beginning of the road leading to thermonuclear energy, does not shadow already the run of study works on the demonstration nuclear plant DEMO. The DEMO Working Group was established in 2010, and in 2012 there was called an Expert Group to prepare a document concerning the Strategic Orientation of the Fusion Program. Both of these bodies have members from Poland (prof. K. Kurzydłowski of WUT, and prof. W. Dominik of UW) [15]. The R\&D communities for fusion in Poland has organized itself into a consortium to establish an R\&D project enabling efficient cooperation with very active European Community gathered in EURATOM organization. Members of the consortium under organization are: IFPiLM, Asociation Polatom, IFJ PAN, NCBJ, AGH, UW and Warsaw University of Technology.

Pragmatic approach to thermonuclear power is based on defining realistic assumptions for demonstration power plant in tight cooperation with industry, and now total concentration of efforts on a single experiment - the ITER. Concentrating of the European fusion laboratories efforts, there was defined a consolidated approach based on eight missions - or strictly defined priorities on the EFRM (European Road Map for Fusion). The required condition to realize the EFRM is providing its strongly innovative character by engaging the industry from the beginning of its realization. The final aim is burdening of the industry with the whole responsibility for the commercial thermonuclear plant, after the potential success of the DEMO infrastructure. DEMO has to fulfill all assumed exploitation conditions. The key element in the success of DEMO is the issue of development of highly resistant neutronic materials. In the area of neutronic and fusion materials it is extremely important that the industry will be constantly engaged, from the very beginning of the EFRM realization. Another component of essence of the EFRM is the ability to confine the costs of building of the infrastructure, first demonstration and next commercial fusion power plants. EFRM assumes strong international cooperation, in such a way, that Europe as a common area might have obtained the necessary knowledge, before the year of 2030 , to start building a demonstration thermonuclear power plant [16].

Pragmatic approach to the thermonuclear power energy requires necessary preparatory steps, combined directly and indirectly with the intensification of the R\&D in this area, as a common effort of many European nations. An important step is to increase the support for teaching and training of new research and technical experts in the field. The following kind of research is assumed to be associated with the EFRM: technical - directly combined with the project, basic associated with the project, basic and applied basic not directly associated with the project but closely related to it. Theoretical research should be run on optimal methods and constituent elements in the project area. Numerical methods associated with the project should be well developed. Especially numerical models of all critical parts of the infrastructure should be prepared and optimized. The research on theoretical and numerical models are essential part of the project.

In this country there is carried on a discussion on the wide participation of national communities in ITER and DEMO [17]. This discussion has to be time correlated with the milestones of the EFRM. Fusion program is a common effort of the whole Europe. Now, a clear choice is as follows: participation in modeling and theoretical calculations and numerical on the measurement data, participation in building of control and measurement systems [16]. This is large contribution, but some communities consider it as too small. Active part of the fusion community in this country considers taking steps to apply to the EU for building in Poland part of the infrastructure of the European scale. Only shared large international research infrastructure existing and exploited in the country adds the local communities to the fusion development club. Such infrastructure like pilot IFMIF facility may be used not only for fusion research, material research but also biomedical, concerning atomic industry, environment protection, energy safety, and many others. Now, the fundamental confinement in these endeavors to build the fusion infrastructure in this country is timing imposed by the EFRM document. Other issues concern finances, political will, strategic decisions in the field of future energy sources, social agreements, determination of research and technical communities, level of research in fusion and related fields like plasma, superconductivity, material engineering and accelerators, etc. [18]-[29].

\section{MISSIONS OF EFRM - BASIC RESEARCH ISSUES TO SOLVE}

EFRM defines eight fundamental issues - theoretical and technical ones, which are necessary to be solved (they are called missions), in order for the fusion technologies to be ripe enough for practical implementations and further consecutive industrialization. The fusion technologies should be dependable, reliable, and of commercial grade. The EFRM missions are defined as follows: 1. Plasma: research on plasma and its properties; areas of stable work for tokomak; 2. Heat: system of heat reception and transfer; 3. Material: research on materials resistant to high neutron fluencies, 4. Tritium: material self-sufficiency; 5. Safety: implementation of safety aspects in fusion environments and infrastructures; 6. Power 
Plant: integrated DEMO project and development of fusion infrastructure; 7. Costs: competitive prices for electricity; 8: Stellarator: new generation of fusion machine - future alternative for tokamak. At the end of each mission we try to estimate the area of participation of experts from this country in the global effort [16].

\section{Plasma}

Obtaining of stable conditions for "burning of plasma" in tokamak depends on many technical conditions. Some of the basic answers to the questions concerning this issue are known, but some are totally unknown. Thermonuclear burning is a dynamic process, taking place in extreme physical conditions, high temperature, high energy, and strong neutronic radiation. One of the unknown answers is to the following question - if it is possible to work over the boundary of threshold plasma density - the Greenwald density limit. Work with plasma of large density may increase the thermonuclear reaction efficiency. However, the basic condition is the work stability of the fuser with thermonuclear burning. The basic issue with DT thermonuclear fuser is its work stability at the conditions where $90 \%$ of the heating energy/power originates from the neutronic radiation (kinetic energy of particles). In these conditions, several kinds of plasma nonstability may be generated. like: ELMs, turbulences, disruptions, etc., which leads to local overheating on the walls of the plasma chamber in the tokamak. It may also lead to the decrease of reaction efficiency. These disturbances are not allowed at all in the exploitation tokomak. Continuous development of effective and fast methods to control these effects is needed, like instabilities in the fast moving plasma stream. Plasma chamber is surrounded by measurement apparatus, sensors, diagnostic systems and actuators, strictly speaking tons of electronics. Now, only a few diagnostic techniques may be applied in the future tokomak. These techniques are now subject to intense development. Building, testing, commissioning and next exploitation of ITER infrastructure should give answers to the questions associated with the work modes of the fuser. Apart from the experiences gathered from the planned biggest tokomak, they are gathered from specialized machines like JET, JT60-SA and a series of small laboratory tokomaks. These supporting machines play an important role in searching the answers to specific technical questions. In the area of plasma research the local community is ready to participate in theoretical investigations, modeling and building of intelligent diagnostic systems of the next generation.

\section{Heat and material}

Reception of immense amounts of heat generated in the DT fuser is now estimated as a fundamental issue number one. If this issue is not be solved satisfactorily, then the thermonuclear power technology in neutronic version may be questioned at all. Large amounts of heat are generated in all fusion reactors with the magnetic trap. Additionally, there are generated reaction products polluting the plasma. The pollutants cause considerable lowering of the efficiency of plasma heating. The EFRM document determines three basic research directions in the range of excess heat removal and particle pollutants from the core of the fuser. Application of a traditional diverter (heat exchanger and plasma cleaner) requires intense radiation cooling and usage of the effect of plasma detachment from the chamber walls. The diverter is a section of the chamber wall which actively receives matter and heat excess. This allows for control of created fusion products and pollutants from the lining of the chamber. The JET and ITER tokomaks have diverters on the bottom of the plasma chamber (torus). There are possible magnetic innovations in the plasma chamber configuration, and new solutions of diverters, like the types of snow flake or Super-X. The aim of these constructions is broadening of the plasma stream in such a way, that the heat generation embraces larger volume, or longer connection is obtained for the diverter and larger radiated power by the diverter. Next conception to solve the reception of the excess heat is application of new materials designed for direct contact with plasma. These may be liquid metals, which take bigger heat loads and may carry the heat away more efficiently than solid state materials. Constructions of classical diverters and modified, innovative ones are tested in smaller laboratory tokomaks, like JET, JT60-SA, MST and in plasma departments of other laboratories. The difference in the scale between the mentioned devices and ITER and DEMO infrastructures is very big and is between 10 and 100, thus, it seems that at least some of the research on dedicated diverters should be done on the target tokomaks, where the geometry and energies are much larger. This is the reason why a special test infrastructure is planned for relevantly large tokomak diverters. The Diverter Tokamak Test Facility DTT is planned as a part of the ITER project. In the research area of the excess heat removal from large tokomaks there are no active experts in this country. The local community may, however, run theoretical and simulation work.

\section{Neutrons}

We do not have now sufficient knowledge on the interaction of high energy neutrons (in this case of kinetic energy around 15 $\mathrm{MeV}, 30 \mathrm{dpa}$ ) with materials relevant for the construction of tokamak components, and exposed to such intense radiation for long time. Several types of these materials are needed. In particular, the materials of first contact with plasma are to be searched and researched, then materials for new diverters, as well as materials converting neutrons' kinetic energy to heat, because this energy can not be directly converted to electricity (as opposed to kinetic energy of fast moving charged particles), etc. It is necessary to build a dedicated neutron test infrastructure called in the EFRM document as the IFMIF (International Fusion Materials Irradiation Facility). Two solutions are predicted: full IFMIF of the cost 1 mld Euro, or reduced or early or pilot IFMIF of the cost 200 mil Euro. The decision on what kind of infrastructure is going to be built and where has to be taken till the end of 2013. One of the questions is if the IFMIF will be a completely new and full infrastructure or reduced based on existing infrastructure but rebuilt and extended. The financial scale is 1:5 in both cases. The IFMIF project is a chance for the local fusion and nuclear 
sciences and technology community, concerning especially the readiness to build a reduced version of IFMIF, using the infrastructure available in the National Center of Nuclear Research in Świerk near Warsaw. Construction of such infrastructure is possible with the support of the European finances. Now the best candidate for IFMIF is accelerator infrastructure based on neutron generation via bombarding with $25-40 \mathrm{MeV}$ deuteron beam of a target made of light ions like lithium or carbon. Neutrons produced in such a source have maximum kinetic energy around $14 \mathrm{MeV}$ and their spectrum is very similar to the DT fusion neutrons.

Building of ITER and next DEMO requires application of a few groups of key and new materials. Not all of them are readily available today. Some of them are not yet available at all. It is necessary to research such materials, find appropriate ones, check thoroughly their properties, and start production. The following groups of materials are of concern: construction materials for tokamak chamber, tokamak framework, etc. These materials have to be resistant to immense heat load, being in direct contact with high temperature and fast moving plasma stream. Materials have to withstand streams of highly energetic neutrons of $14 \mathrm{MeV}$ of big intensity and for long time. In particular these materials have to be resistant to erosion - so as not to pollute fast stream of the fusion plasma. Apart from the above conditions, which are difficult to fulfill by a universal enough material, these materials do not have to be subject to strong neutron excitation/activation, and should be vulnerable to large scale industrial production. Demand for such materials may increase abruptly with the development of atomic industries. Among the materials for the first wall there are considered: boron carbide, tungsten carbide - borium, graphite, composites with carbon fibers, tungsten, molybdenum, multilayer structures made of these materials, etc. JET plasma chamber is lined, since 2009 with beryllium (which replaced graphite) in order to test the solutions predicted for ITER. Tungsten is used for the diverter in JET as well as in ITER. These solutions have still a series of drawbacks, which have to be solved in the near future, if the fusion reactor is to be made efficient enough.

In the research area of intense neutron radiation and materials resistant to such radiation, there is in this country a narrow group of experts, which may add their knowledge actively to relevant proposition of the fusion neutron research project. The possibilities to participate in the project are not confined only to the theoretical and numerical work, like extrapolation and modeling of transposition ITER - DEMO machines. It seems to be possible to create in the Maria Nuclear Reactor in Świerk a laboratory of reactor based fast neutrons: via the inter-core converter of thermal neutrons to fast $14 \mathrm{MeV}$ neutrons; building installation for irradiation of prototype components and devices for ITER in a profiled spectrum of fast neutrons; adaptation of the $14 \mathrm{MeV}$ neutron source to the research on radiation hardness of diagnostic apparatus for ITER.

\section{Tritium}

Radioactive isotope of hydrogen is a strategic material, similar to uranium, under strict control. It is necessary in ITER and DEMO tokomaks to run the DT reaction. There are possible a few solutions, either separate production of tritium, or associated with the fuser. Associated production lowers considerably the risks combined with separate tritium production. In association with these issues, the EFRM predicts the necessity to undertake and run research works on alternative methods of tritium production. These include, for example: cooling the fuser with water with LiPb, or WCLL method, which stands for water cooled LiPb blanket. Now, there are considered methods using helium coolant. There are also considered cooling systems with double blanket $\mathrm{LiPb}$ and $\mathrm{He}$, in a configuration called a dual cooled tritium breeding blanket PPCS. There are researched alternative cooling methods to He. The research project is associated with providing tritium selfefficiency for DEMO, and is intensely developed at the ITER infrastructure. ITER has a separate test cooling blanket module TBM. It works in the configuration of $\mathrm{LiPb}$ cooled with $\mathrm{He}$ (HCLL - He cooled LiPb blanket) and in configuration of stone bed cooled with He (HCPB - He cooled pebble bed).

This country has no active experts in the domain of cooling large infrastructures with strong neutron radiation. It is possible, however to create in this country the research tritium laboratories (prof. U. Woznicka, IFJ PAN).

\section{Safety}

There is a number of common factors concerning safety in the whole atomic industry. There are also characteristic safety factors for the thermonuclear fusion environment. A positive aspect is that many safety factors associated with the nuclear power based on heavy fission fuel is minimized in thermonuclear power with light fusion fuel. The research on the safety of fusion technologies of energy production are carried out to obtain relevant safety certificates - licenses (for design, for building and for exploitation) by the future thermonuclear plant. This concerns initially the DEMO plant and next commercial plants. ITER obtained French and European safety licenses for building thermonuclear infrastructure and doing fusion research. The aim of ITER is neither production of energy, nor electrical, nor any energy at all. The aim of ITER is testing of the foundations of thermonuclear fusion and proving the principle. However, the experiences gathered during the process of the European legalization and licensing of the infrastructure and actions and methods on the infrastructure are invaluable to extend this process on the future infrastructure of DEMO. Long lasting and very precise licensing process for ITER confirmed relative safety of the nuclear fusion, and indicated essential research area. This research will have influence on the safety license for the commercial thermonuclear plant.

There were listed three areas which differ both large infrastructures ITER and DEMO. These are: tritium, neutrons and radioactive materials. DEMO will require more massive tritium economy, including in this tritium flow in continuous reaction and its stock. The reactor in DEMO infrastructure generates much bigger neutronic stream to the reactor blanket than ITER. The whole DEMO infrastructure will have to manage efficiently considerable amounts of radioactive materials. Effective methods have to be mastered to remove tritium from the nuclear waste. Radioactive waste materials 
have to be neutralized via transmutation. ITER, and especially DEMO have to manage safe keeping of waste radioactive materials.

\section{Thermonuclear plant}

DEMO infrastructure is expected to be a full featured test thermonuclear power plant, effectively producing electrical energy, at certain cost. It should not be a repetition of the fully experimental infrastructure of ITER. Obviously, DEMO should and has to base on ITER experiences. During the first period defined by EFRM, or during the Horizon2020, the DEMO receives nearly no financing. These are only small investments in works on superconducting magnets, devices for plasma heating and maintaining the heating current, vacuum chamber and pump system, diagnostic devices and remote control. These investigations, usable for DEMO, are however used for the development of ITER. During H2020, there is predicted work on a standard document CDR - the Conceptual Design Report/Activity. Intensification of investments, directly for DEMO is predicted after the initial period of exploitation of ITER. This is a decade of 2020-2030, during which there will be prepared next engineering document EDA Engineering Design Report/Activity. Necessary element of the DEMO project realization will be preparation of new diagnostic techniques, because many of existing techniques will not be subject to scaling due to completely different work conditions of systems in much more adverse environments than ever before. This area of research is also a chance for the local communities in this country.

\section{Costs}

Costs of the fusion program are very delicate matter. One of the aims of the ITER, and in particular the DEMO, initiatives is to prove that the costs of energy production by fusion method are competitive to other methods, actually all methods including classical ones, coal or crude oil electricity, from renewable energy sources, from alternative energy sources, etc. If this proof fails then the fusion energy has less sense than predicted. Precise and thorough research on fusion energy are very costly. But investing in them now is the only way to check the feasibility in the future. Actually, these investments are more devoted to future energy sources than to the development of fusion science. Of course, no one doubts, that fusion science gains also a lot. The clear aim are, however, commercial and fully long term exploitation grade fusers. Any evaluations are impossible without realization of the very complex and expensive ITER and DEMO experiments. The investment costs of the project, as defined by the EFRM, covered in common by the EC and member countries of the consortium, are around 500 million Euro, slightly approximated up, per annum, in the whole period of 2013-2020, and probably till 2050.

\section{Stellarator}

The ideas to realize thermonuclear fusion are subject to constant development. EFRM positions the stellarator development as a possible future alternative for tokomaks. A stellarator of Helias type W7-X is under construction in Greifswald.
It is one of the European priorities for H2020. Other types of stellarators, determined by the shape of toroidal-solenoidal magentic trap are: Torsatron, Heliotrop, Helias and Heliac. The beginning of the exploitation of European stellarator is predicted for 2015. W7X will work in the quasi CW mode. Maximal time of continuous work is designed for about half an hour. Future stellarators are predicted for fully continuous work. Stellarator idea originates from 50ties of XX century. Better fusion results obtained in tokomaks delayed their development for long time. End of XXtieth century saw return to this idea, as a hope for avoiding the issues of fast excess neutrons generated in DT tokomaks, as well as simplification and cheaper solution of the fuser core. Tokomaks provide necessary bending of the magnetic field lines not by the shape of the solenoidal - toroidal magnetic trap, but via the current flowing in the heated plasma. The lines of magnetic field around the flowing current in plasma connect with the toroidal field, creating the resulting helical field which wraps around the torus in both directions. Stellarator also has toroidal field, but has no azimuthal symmetry, as tokomak. Stellarator has a discrete rotational symmetry. Most frequently it is a regular pentagonal symmetry. Stellarator does not require toroidal current, what greatly simplifies its construction. Stellarators, at the current stage of development, have many drawbacks like: more difficult construction of diverter, more complex modeling of plasma geometry, necessity to apply 3D solenoids of complex geometry, etc. Stellarator development goes into direction to obtain quasi symmetric magnetic field, as in the HSX machine - Helically Symmetrical Experiment, which is tested at Uni. Wisconsin. Potential participation of the local community in stellarator development is large.

\section{EUROPEAN FUSION - INTERNATIONAL COOPERATION}

It is obvious, that other advanced regions of the world are working on their own fusion development and future implementation programs. Inside such a cooperation, with these regions, the EFRM predicts close cooperation with fusion energy initiatives also outside Europe. The subjects already realized in international cooperation are: common exploitation of JT-60SA infrastructure in cooperation with Japan to prepare the second development phase of ITER; construction of infrastructure pilot-IFMIF (early neutron source) also in cooperation with Japan inside the framework of advanced phase of EVEDA experiment; cooperation on common international project of test laboratory for tokamak diverters; cooperation with tokamak infrastructures CFETR in China (Chinese Fusion Experimental Tokamak Reactor), and FNS neutron infrastructures in USA (Fusion Neutron Science); sharing the know-how concerning the program TBM; usage of experimental spallation reactors outside Europe; cooperation with stellarator infrastructures other than HELIAS (for example Heliotrons and Compact Stellarators). The European Union offers international partners a cooperation at JET tokamak, as a place for tests for solutions for ITER. The EFRM predicts co-financing of partners participation in international fusion infrastructures. 


\section{Realization of Fusion RoAd Map in EuRope}

Now, in the European fusion communities lasts a vivid discussion on the EURATOM program for 2014-2020. The new program, just presented by the European Commission, resigns from realized till now association contracts and assumes a large intensification of activities in Europe on fusion. There is created a new financing mechanism of common actions, called the Action of Co-Financing. The mechanism assumes common financing of particular undertakings by the EC and partners - co-owners of the built and/or modernized fusion infrastructure. Realization of the whole EURATOM Program in the fusion domain is done by a single Consortium, created on the basis of the Association. Consortium Coordinator is Max Planck Institute of Plasma Physics in Garching (IPP). A duty of the Consortium will be presentation of the plan for 2014-2020 in the EURATOM Program. The project will be reviewed by a nondependent group of experts. After setting down the positions, the EC will sign, with the Consortium, a standard document Grant Agreement for realization of the co-financed project. Financial input may be in-kind or in cash. The Consortium is responsible for the scientific realization of the project. Membership countries are represented in the Consortium by single liaisons (representatives of institutions with fusion infrastructure) nominated by particular governments.

\section{Future of Fusion RESEARCH IN EUROPE AND POLAND}

The idea to build a large European research infrastructure in a form of a strong neutron source, meets in this country a large interest in the local fusion community. Industry in this country should actively participate in this large European project of fusion energy. It is necessary to predict, in these conditions, other applications of the strong neutronic radiations fields, than only for the fusion materials. This would lead to bigger interest among related Polish research communities in such areas as electronics and telecommunications, photonics, nuclear physics, biomedicine, atomic industry, etc. The European Fusion Project is another chance for this country to apply for very large European research infrastructure. National fusion community should apply for financing of a part of this project from the National Applied Research Funding Agency NCBiR along with application in Europe via the Euratom Agency. The effort should go in the direction of checking the possibility to build in this country so called Early IFMIF Neutron Infrastructure (pilot IFMIF). It is necessary to extend the cooperation of researchers from this country at the construction of the European Stellarator W7-X. Fusion community should determine the local road map for Polish Working Groups participating in the EFRM, taking into account only partial reimbursement of the costs.

\section{CONClusions AND ACKNOWLEdGments}

The European Road Map for Fusion is a very important undertaking having long lasting consequences for the local science, development and technology, in the European scale and in local national scales. ITER, DEMO, DDTF, IFMIF and other related infrastructures will change the European fusion landscape forever. These infrastructures will require a lot of investments and research efforts in material engineering, photonics and electronics, mechanical engineering, large project management etc. It will engage several hundreds, if not thousand European researchers for several decades. Polish scientists will participate in these efforts actively.

The author would like to thank some members of the Polish Fusion Community for cooperation and additional information. This subject was debated also during a separate topical session at the Wilga 2013 Symposium on Electronics for HEP Experiments, FELs, Fusion and Astroparticle Physics [7].

\section{REFERENCES}

1] European Fusion Development Agrement - EU-EFDA: www.efda.org

[2] FuseNet - The European Fusion Education Network: fusenet.eu.

[3] The European Atomic Energy Community - EURATOM: www.euratom.org.

[4] Culham Centre of Fusion Energy, Joint European Torus: www.ccfe.ac.uk/JET.aspx.

[5] ITER web page: www.iter.org.

[6] T. Matulewicz et al., "The Future of Fusion Research in Poland," in Fusion Conference IFD UW, Warsaw, May 2013.

[7] WILGA 2013 Symposium on Electronics for High Energy Physics Experiments: wilga.ise.pw.edu.pl.

[8] R. S. Romaniuk (editor), "Photonics applications in astronomy, communications, industry, and high energy physics experiments 2013," Proceedings of SPIE, vol. 8903, 2013.

[9] R. S. Romaniuk, "Fuzja - perspektywa 2050," Elektronika, vol. 54, no. 6, pp. 73-78, 2013

[10] IFMIF Web page: www.ifmif.org.

[11] National Ignition Facility NIF-LLNF: lasers.llnl.gov.

[12] Laser Inertial Fusion Energy LIFE: life.llnf.gov.

[13] Spallation Neutron Source SNS: neutrons.ornl.gov.

[14] European Spallation Source ESS: europeanspallationsource.se.

[15] R. Zagórski, "Fusion Road Map, Program of fusion research in Poland," in Fusion Conference. Warsaw: IFD UW, May 2013.

[16] U. Woźnicka, "Fusion Electricity: A roadmap to realisation of fusion energy," in Fusion Conference. Warsaw: IFD UW, May 2013.

[17] Foresight for thermonuclear energy: [energetykatermojadrowa.pl].

[18] R. S. Romaniuk, "Photonics applications and Web engineering: Wilga May 2013," Proceedings of SPIE, vol. 8903, 2013, art. no. 890303.

[19] _ _EuCARD2 - enhanced accelerator R\&D in Europe," Proceedings of SPIE, vol. 8903, 2013, art. no. 89031P.

[20] - "Accelerator science and technology in Europe - EuCARD 2012,' Proceedings of SPIE, vol. 8454, 2012, art. no. 84540 O.

[21] — , "Accelerator technology and high energy physics experiments, Photonics Applications and Web Engineering, Wilga May 2012," Proceedings of SPIE, vol. 8454, 2012, art. no. 845404.

[22] _ - "Photon physics and plasma research, Photonics Applications and Web Engineering, Wilga May 2012," Proceedings of SPIE, vol. 8454, 2012, art. no. 845405

[23] — "Accelerators for society: succession of European infrastructural projects: CARE, EuCARD, TIARA and EuCARD2," Proceedings of SPIE, vol. 8903, 2013, art. no. 890320.

[24] — "Visions of the future of particle accelerators," Proceedings of SPIE, vol. 8903, 2013, art. no. 890324.

[25] — "Accelerator science and technology in Europe 2008-2017," Proceedings of SPIE, vol. 8903, 2013, art. no. 89031P.

[26] _ - "Accelerator infrastructure in Europe EuCARD 2011," International Journal of Electronics and Telecommunications, vol. 57, no. 3, pp. 413-419, 2011.

[27] R. Romaniuk, "EuCARD 2010 accelerator technology in Europe," International Journal of Electronics and Telecommunications, vol. 56, no. 4 , pp. $485-488,2010$.

[28] — "Advanced photonic and electronic systems Wilga 2010," International Journal of Electronics and Telecommunications, vol. 56, no. 4 pp. 479-484, 2010.

[29] R. S. Romaniuk and K. Pozniak, "Metrological aspects of accelerator technology and high energy physics experiments," Measurement Science and Technology, vol. 18, no. 8, 2008, art. no. E01. 